\title{
A MODEL OF THE INJECTION MOULDING PROCESS
}

\author{
J. WHALE, N. FOWKES, G. HOCKING ${ }^{1}$ and D. HILL
}

(Received 10 September 1992; revised 30 April 1994)

\begin{abstract}
This paper is concerned with the injection moulding process, in which hot molten plastic is injected under high pressure into a thin cold mould. Assuming that the velocity and temperature profiles across the mould maintain their shape, a simple steady state model to describe the behaviour of a Newtonian fluid during the filling stage is developed. Various phenomena of the process are examined, including the formation of a layer of solid plastic along the walls of the mould, and the relationship between the flux of liquid plastic through the mould and the average pressure gradient along the mould. In any given situation, it is shown that there is a range of pressures and injection temperatures which will give satisfactory results.
\end{abstract}

\section{Introduction}

The injection moulding process is one of the most important operations involved in polymer processing. The mould usually consists of two halves which are brought together, clamped into position and kept at a constant temperature. Hot molten plastic is then forced under pressure into the cooler mould. After the plastic has solidified, the clamps are released, the moulded object is ejected and the cycle is repeated. In this way, objects ranging in size from toy building blocks to car bonnets are produced.

Historically, those involved in injection moulding have relied on a combination of experience and a few rules of thumb to produce reasonably successful results. However, there are two important factors which have combined to make the traditional approach outdated. Firstly, manufacturers realize they can achieve lower production costs by having shorter cycle times and reduced object weight. Secondly, there are now attractive markets for large injection mouldings such as business machine panels, various panels for the car manufacture industry and even complete boat hulls and decks. These considerations have led to quality control problems, since the items

Department of Mathematics, University of Western Australia, Nedlands WA 6009, Australia.

${ }^{1}$ School of Mathematical and Physical Sciences, Murdoch University, Murdoch WA 6150, Australia.

(C) Australian Mathematical Society, 1995, Serial-fee code 0334-2700/95 
are prone to cracking and buckling.

To avoid such problems, it is desirable to be able to predict the pressures, temperatures and times required to quickly mould 'acceptably imperfect' objects. Many of the items to be moulded have a thin geometry, and special difficulties are often experienced in using this process for such objects [1,7]. Improvement in both the accuracy and computational efficiency of the numerical simulations of the process are desired and will result only from a better understanding of the physics and mathematics of the melt flow behaviour and buckling process. In this paper, we will attempt to improve the understanding related to the process for situations involving such thin geometry.

In the process, molten plastic is injected into the mould through a 'gate'. Liquid plastic in contact with the mould freezes immediately, thereby partially insulating the liquid plastic from the cold mould walls. The solidified plastic layer continues to build up during the mould filling stage. Molten plastic is extremely compressible so that after the mould is 'full', up to $20 \%$ more plastic can be forced into the mould until pressures equilibriate and the gate 'closes'. This phase is called the compensation phase or packing stage of injection moulding [6] . Molten plastic shrinks as it solidifies so such compensation is desirable. After complete solidification the moulded item is ejected and deforms into a shape determined by the stresses 'frozen into' the item during the moulding process. This shape is one that minimizes the elastic strain energy and so, in principle, can be determined from a knowledge of the 'frozen in' spatial distribution of the elastic properties.

These local elastic properties are determined by the local orientation of the long chain plastic molecules. For example, plastic molecules will be aligned if they freeze under high shear conditions (for example, during the injection phase) and the resulting material is characterized by a small Young's modulus in the direction of alignment. Plastic molecules which freeze under low shear conditions (for example, during the compensation phase) will be randomly orientated, and consequently will have elastic properties which are independent of direction. Thus, to determine the final shape of the object, it is necessary to investigate the growth (and stress environment) of the solid plastic skin within the mould during the moulding process. If high enough temperatures and pressures are employed, buckling is not a problem, and manufacturers and research workers have avoided the buckling problem by working in this range. However, high temperatures mean longer cycle times and again economic pressures force one to consider the difficult questions posed above.

In the absence of solidification, the physics of this type of flow through narrow channels is well understood. Ockendon [8] follows approximations made by Pearson [9] in developing asymptotic methods to analyse the flow and, in particular, the phenomenon of thermal runaway, which occurs when injecting a fluid with temperature-dependent viscosity into a narrow channel. In this situation, the heat generated causes both an increase in shear and a decrease in viscosity. If the increase in shear dominates 
the viscosity decrease, temperature levels continue to rise and may run away to very high levels. Elliott and Ockendon [4] and Tayler [12] consider the injection moulding process as an application of Hele-Shaw flow between slightly separated parallel plates.

The filling of the mould is treated by Wang et al. [13] in an effort to develop an integrated computer program incorporating a numerical simulation of mould dynamics. Wang [14] presents some accomplishments of the subsequent research. Further computer-aided applications have been developed where the filling stage has been simulated by means of the Hele-Shaw equations for non-Newtonian flow [3], [2]. Recently, attempts have been made to develop simplified simulation models [5].

In 1986, the Australian Mathematics in Industry Study Group [1] was approached to develop a model which contained the essential features of the problem but did not rely on any significant computing. In particular, the Group was interested in the formation of the solid plastic skin on the walls of the cooled mould and its effect on melt flow behaviour. They examined the steady state solid plastic skin profile as a function of distance from the gate for a Cartesian steady state model with thin slab geometry. The assumption that the profiles of fluid velocity and temperature preserve their shape along the length of the mould allowed them to obtain approximate solutions for the case of a Newtonian fluid. This paper also deals with a steady state model, and is concerned with the growth of solidified plastic during the mould-filling stage and in particular its influence on the flux-pressure relationship within the mould.

\section{The steady state problem}

There are a number of difficulties in attempting to solve the full problem for this physical process, such as complex and possibly thin geometries (many moulded items are millimetres thick and many centimetres in length), the change in state, and the many time scales involved in the full procedure. In many cases the flow is also non-Newtonian. Our goal, however, is to develop a simple model of the actual flow behaviour during the filling stage, and consequently we consider the simplest problem possible which still contains the essential features of the process. This corresponds to examining plane flow through an open ended channel (see Figure 1).

We assume the plastic is incompressible throughout the filling stage and the mould is of finite length $L$ and half-width $H_{0}$ where $H_{0} \ll L$. A pressure difference $p_{0}-p_{L}$ is applied between the ends of the mould forcing a volume flux $Q$ through the mould. The solid plastic skin forms on the walls of the mould reducing the channel width for flow of liquid plastic. For a given position determined by longitudinal co-ordinate $x$, the half width of the channel is $H(x)$ so that the solid sidewall skin has width $H_{0}-H(x)$. The fluid is injected with entry temperature $T_{0}$ while the mould walls are maintained at a constant temperature $T_{w}$. The solid-liquid interface is at the 


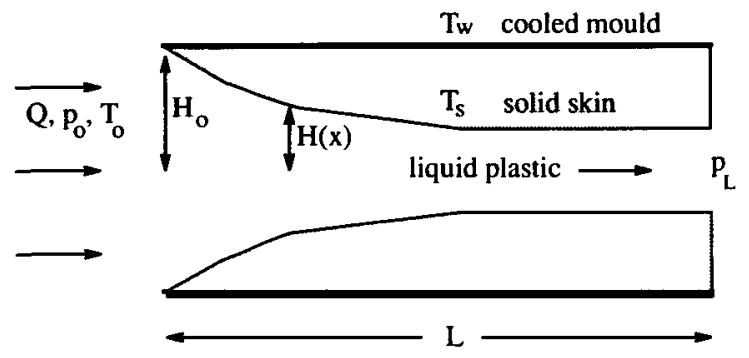

FIGURE 1. Definition sketch of the problem modelled in this paper.

solidification temperature $T_{s}$ and we have $T_{0}>T_{s}>T_{w}$ (see Figure 1).

To avoid latent heat complications we concentrate on a steady state situation. The steady state model is appropriate only during the filling stage and is convenient for understanding the major aspects of the physics of the problem. Plastic is driven into the channel (and drawn out the other end) by a fixed pressure difference and we assume sufficient time has elapsed so that all transients have settled down. In an infinite mould, either the skin thickness reaches an equilibrium level at which viscous heat generation within the plastic balances heat loss to the mould, or the mould closes off the gap. In a finite mould, the solid layer maintains the same shape (time invariant) but may or may not attain a constant thickness. Note, then, that we have an important distinction between steady state and equilibrium.

Exploiting the thin geometry, we obtain the standard lubrication theory approximations for the equations of mass, momentum and energy conservation as:

$$
\begin{aligned}
\int_{0}^{H(x)} u(x, y) d y & \left.=\frac{Q}{2}, \text { (independent of } x\right) & & \text { Continuity } \\
p_{x} & =\left(\mu u_{y}\right)_{y}, & & x \text { momentum } \\
p & =p(x), & & y \text { momentum } \\
\rho c\left(u T_{x}+v T_{y}\right) & =k T_{y y}+\mu u_{y}{ }^{2} . & & \text { Energy }
\end{aligned}
$$

The following boundary conditions must also be satisfied:

$$
\begin{aligned}
& u=v=0 \quad \text { on } y=H(x), \\
& u_{y}=T_{y}=0 \quad \text { on } y=0
\end{aligned}
$$

and

$$
-k T_{y}(x, H(x))=k_{s}\left(\frac{T_{s}-T_{w}}{H_{0}-H(x)}\right) \quad \text { on } y=H(x)
$$


Since we don't know the thickness (as a function of $x$ ) of the solid sidewall skin beforehand, the equations are unmanageable using exact methods. Austin and Miller [1] arrived at a system that is amenable to solution by assuming the velocity and temperature profiles are shape preserving. That is, they may be written as a product of a scaling function which varies with position $x$ and an $\mathrm{O}(1)$ profile function that gives the detailed shape and depends only on $y / H(x)=\xi$, that is,

$$
u(x, y)=\hat{u}(x)[1-\Phi(\xi)], \quad T(x, y)=\hat{T}(x)[1-\Psi(\xi)]+T_{s} .
$$

This assumption of shape preservation is in the spirit of Kármán-Pohlhausen (see [10]), the intention being to encompass the essential features of the problem. The shape-preserving form for $u$ can actually be found from (2.2) and (2.6) if $\mu$ is constant, and it turns out to be

$$
\hat{u}=-p_{x} H^{2} / 2 \mu, \quad \Phi(\xi)=\xi^{2} .
$$

The form for $\hat{T}$ is not in general exact, but it is a useful rough approximation, as is that for $u$ if $\mu$ is not constant.

The interface boundary condition (2.5) means that $\Phi(1)=\Psi(1)=1$ whilst the symmetry condition (2.6) implies that $\Phi^{\prime}(0)=\Psi^{\prime}(0)=0$.

\section{The model equations}

A tractable system is obtained by integrating the lubrication theory equations over the half thickness $0<y<H(x)$ to obtain ordinary differential equations in $x$. The profile shapes give $O(1)$ constants when integrated. In [1], it is established that the qualitative character of the solution is essentially independent of the particular profile, the only difference being these $O(1)$ constants.

Since we do not attempt to determine the correct velocity profile, it is necessary to introduce effective plastic properties, denoted with a subscript $E$. These effective parameters are the true parameters multiplied by $\mathrm{O}(1)$ constants which depend on the assumed profile shape.

Performing the half thickness integrations and using (2.8) results in the equations

$$
\begin{aligned}
\hat{u}(x) H(x) & \left.=Q_{E}, \text { (independent of } x\right) & & \text { Continuity } \\
-\frac{d p_{E}}{d x} & =\mu_{E} \frac{\hat{u}}{H^{2}(x)}, & & x \text { momentu } \\
\rho_{E} c Q_{E} \hat{T}^{\prime}(x) & =-k_{E} \frac{\hat{T}(x)}{H(x)}+\mu_{E} \frac{\hat{u}^{2}(x)}{H(x)} & & \text { Energy }
\end{aligned}
$$

and the boundary condition (2.7) yields

$$
k_{E} \frac{\hat{T}(x)}{H(x)}=k_{s}\left(\frac{T_{s}-T_{w}}{H_{0}-H(x)}\right),
$$


where the effective parameters are given by

$$
\begin{gathered}
Q_{E}=Q / C_{1}, \quad \mu_{E}=C_{2} \mu, \\
p_{E}=\left(C_{2} / C_{3}\right) p, \quad k_{E}=C_{4} k, \quad \rho_{E}=C_{5} \rho,
\end{gathered}
$$

where

$$
\begin{gathered}
C_{1}=2 \int_{0}^{1}[1-\Phi(\xi)] d \xi, \quad C_{2}=\int_{0}^{1}\left[\Phi^{\prime}(\xi)\right]^{2} d \xi \\
C_{3}=\Phi^{\prime}(1), \quad C_{4}=\Psi^{\prime}(1), \quad C_{5}=\int_{0}^{1}[1-\Phi(\xi)][1-\Psi(\xi)] n d \xi .
\end{gathered}
$$

If the viscosity is constant, (2.9) and (3.3) can now be used to obtain $\hat{T}$ in terms of parabolic cylinder functions. However, since it is intended to develop an approach that will prove useful for the analysis of situations in which the viscosity is shear and/or temperature dependent the general profile forms (2.8) will be retained.

For simplicity we drop the accents from the profile terms $\hat{u}(x)$ and $\hat{T}(x)$ and the subscripts from the six effective parameters.

The energy equation (3.3) governs the horizontal transport of heat within the liquid plastic. The first term on the right of the equation represents heat being lost from the liquid plastic to the solid plastic. The second term on the right represents heat being gained by the liquid plastic via viscous heat generation due to shear. The solid heat conduction equation (3.4) governs the lateral transport of heat. Heat is conducted from the liquid plastic to the solid plastic and passes through to the cold mould.

We also have an injection condition given by

$$
T(0)=T_{0},
$$

where $T_{0}$ is the temperature of the plastic as it is injected into the mould.

Introducing the scalings

$$
H(x)=H_{0} h(x), \quad x=L X, \quad T(x)=T_{0} \tau(x),
$$

the energy equation (3.3) becomes

$$
-\tau_{X}=\alpha\left(\eta \frac{\tau}{h}-\frac{\epsilon}{h^{3}}\right)
$$

where $\alpha=\frac{L}{H_{0}}$ is the aspect ratio and $\eta$ and $\epsilon$ are dimensionless groups

$$
\eta=\frac{k}{\rho c Q}, \quad \epsilon=\frac{\mu Q}{H_{0}^{2} \rho c T_{0}}
$$


Similarly, the boundary condition (3.4) becomes

$$
\tau(X)=\frac{\beta h(X)}{1-h(X)}
$$

where

$$
\beta=\frac{k_{s}\left(T_{s}-T_{w}\right)}{k T_{0}} .
$$

The injection condition (3.7) becomes $\tau(0)=1$ and (3.11) can then be used to write $\beta$ in terms of the initial half-width of the channel

$$
h(0)=1 /(1+\beta) .
$$

It is clear then that there exists a relationship between the injection temperature and the initial thickness of the solid plastic in the channel. This seems strange since we might expect to be able to specify any temperature at injection, and also have zero solid skin thickness at the injection point $h(0)$. This problem arises because of the lubrication theory approximations that have been made. We reconcile this by placing the $y$-axis not actually at the start of the mould but a small distance into it.

Using (3.11), we eliminate $\tau$ from (3.9) to yield an ordinary differential equation for the varying channel width due to solidification,

$$
h_{X}=\alpha \eta\left[\gamma \frac{(1-h)^{2}}{h^{3}}+h-1\right]
$$

where

$$
\gamma=\epsilon /(\eta \beta) .
$$

The equilibrium width of the channel $h_{m}$ occurs when $h_{X}=0$. Equation (3.14) gives

$$
\gamma\left(1-h_{m}\right)=h_{m}^{3} .
$$

We note that $\gamma$ governs the magnitude of the equilibrium thickness and $\eta$ determines how far down the length of the mould the equilibrium thickness occurs. This is so because

$$
\gamma=\frac{\mu Q^{2}}{H_{0}^{2} k_{s}\left(T_{s}-T_{w}\right)}
$$

represents a ratio of viscous heat generation to transverse heat loss through the solid to the walls, while

$$
\eta=k /(\rho c Q)
$$

is a ratio of conductive heat loss to the mould walls over the heat supplied through entry. 
Figures 2 and 3 graphically illustrate the importance of the two dimensionless parameters. Figure 2 clearly shows the effect that increasing $\gamma$ has on the channel width, and Figure 3 the effect that increasing $\eta$ has on the distance down the mould at which the equilibrium is reached.

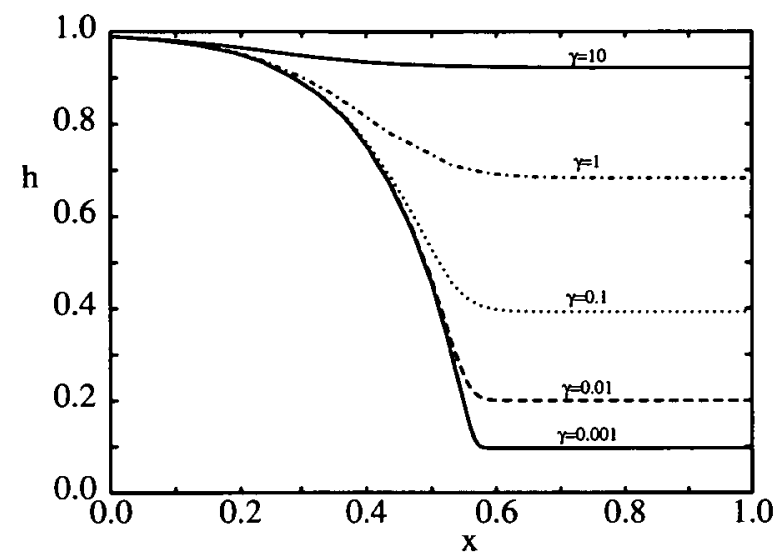

FIGURE 2. Diagram showing the effect of varying $\gamma$ on the skin thickness. In the situation shown, $\eta=0.04, \alpha=200$ and $\beta=0.01$. Larger values of $\gamma$ reduce the equilibrium thickness of the solid skin.

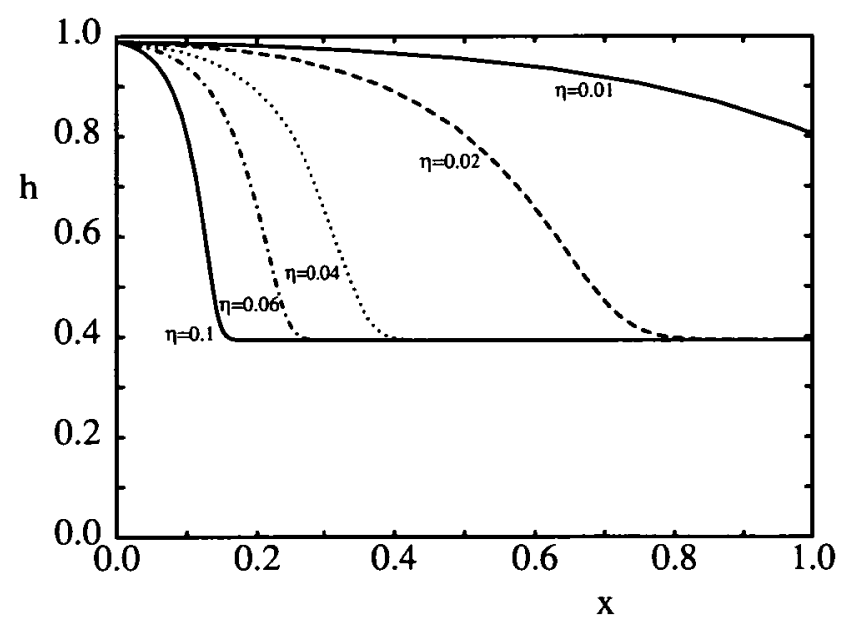

FIGURE 3. Diagram showing the effect of varying $\eta$ on the distance down the mould at which the equilibrium is reached. In the situation shown, $\gamma=0.1, \alpha=300$ and $\beta=0.01$. Larger values of $\eta$ reduce the distance taken for the equilibrium thickness to be reached. 


\section{The flux-pressure relationship}

Let us now investigate the influence of the solid sidewall skin on the flux-pressure relationship within the mould. In particular we would like to compare the results obtained with those for a channel where solidification does not take place.

The continuity and momentum equations of the model combine to give

$$
-p_{x}=\mu Q / H^{3}(x) \text {. }
$$

The volume flux, $Q$ is independent of $x$, and so integrating along the length of the mould yields

$$
Q=\frac{p_{0}-p_{L}}{\mu}\left[\int_{0}^{L}[H(x)]^{-3} d x\right]^{-1}
$$

Defining

$$
P_{0}=\left(p_{0}-p_{L}\right) / L
$$

and employing the previous scaling, along with

$$
Q=Q_{0} q,
$$

we obtain

$$
q=\left[\int_{0}^{1}[h(X)]^{-3} d X\right]^{-1}
$$

if $Q_{0}$ is such that

$$
Q_{0}=P_{0} H_{0}^{3} / \mu .
$$

Note that (4.6) is the flux-pressure relationship for the nonsolidification case, and gives us a prototype example with which to gauge the effect of solidification on the flow in the mould.

If we now define

$$
\gamma_{0}=\frac{\mu Q_{0}^{2}}{H_{0}^{2} k_{s}\left(T_{s}-T_{w}\right)}=\frac{P_{0}^{2} H_{0}^{4}}{\mu k_{s}\left(T_{s}-T_{w}\right)}
$$

and

$$
\eta_{0}=\frac{k}{\rho c Q_{0}}=\frac{\mu k}{\rho c P_{0} H_{0}^{3}},
$$

the ordinary differential equation (3.14) for the thickness of the channel becomes

$$
h_{X}=\alpha \eta_{0}\left[\gamma_{0} \frac{(1-h)^{2}}{h^{3}} q+\frac{h-1}{q}\right] \text {. }
$$

After careful examination, it becomes apparent there is more than one approach to solving the equations and finding the flux-pressure relationship. 
In practice, for a mould of fixed length, we apply a certain pressure difference between the ends of the mould and ask, what is the resulting flux of liquid plastic through the channel? Adopting this procedure, we set a value for $P_{0}$, the average pressure gradient, and calculate the parameters $\gamma_{0}$ and $\eta_{0}$. To determine the unknowns $h(X)$ and $q$, requires us to simultaneously solve the integral equation (4.5) coupled with the differential equation (4.9), subject to the injection condition (3.13). The flux of liquid plastic through the mould is then found by substitution of (4.6) into (4.4).

However, if we decide on a particular flux $Q$ first, (4.5) and (4.9) decouple. For a given $Q$, the nondimensional parameters $\gamma$ and $\eta$ can be evaluated. An explicit solution for $h(X)$ is obtained from solving (3.14) in accordance with (3.13). Then $q$ is determined via (4.5) and again we substitute (4.6) into (4.4) to find the pressure drop across the mould. We choose to use this latter 'fixed flux' approach. The ordinary differential equation (3.14) was solved for a range of parameter values using a fourthorder Runge-Kutta scheme from one of the many available packages, and then $q$ was obtained using the trapezoidal rule.

Before we consider the results, let us consider the situation in which the solid layer attains a constant thickness. As $X$ increases, $h$ changes from $h(0)$ to an equilibrium value $h_{m}$. We have equilibrium whenever heat gained by the liquid via viscous generation balances conduction loss to the cooled mould walls.

The appropriate terms of equation (4.9) are

$$
\begin{aligned}
& \text { Loss }=\frac{k_{s}\left(T_{s}-T_{w}\right)}{H_{0}(1-h)}, \\
& \text { Gain }=\left(\frac{\mu Q^{2}}{H_{0}^{3}}\right) \frac{1}{h^{3}} .
\end{aligned}
$$

Plotting the two functions (see Figure 4), $h_{m}$ is determined from their point of intersection. Naturally $h_{m}$ is an increasing function of $Q$. We find that this point is unique and moreover, analysis of the sign of $h_{X}$ in the vicinity shows the equilibrium to be stable, so that for a given flux, the equilibrium will be reached whether we start with a very narrow entry channel or a very wide one. Due to the approach we have adopted, this is to be expected. If the channel has a particular flux going through it then it must stay open and steady solutions always exist. The pressure drop required to drive the flow, however, may be completely unrealistic.

Therefore, for any starting value of $h(0)$ we approach equilibrium. There are two possible cases. If $h_{0}>h_{m}$, the liquid plastic cools and the channel narrows as it converges to its equilibrium height. If $h_{0}<h_{m}$, we have a situation where we are pushing the liquid plastic through a narrow entrance. In this case, the shear is so large that the heat gained by the fluid overrides the heat lost to the mould walls and the channel expands. 


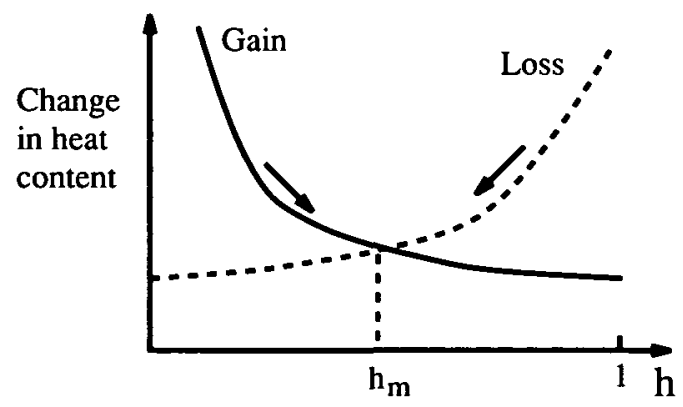

FIGURE 4. The loss and gain terms of (4.9). The direction of the arrows gives the behaviour of $h$ as $X$ increases. Thus $h_{m}$ is a stable equilibrium.

\section{Results}

There are of course an almost infinite number of possible 'experimental' examples which we could consider, involving different values of the various parameters. However it is not our intention to provide such a catalogue of possible outcomes, since we are working with a very simple model. Instead we are interested in considering the qualitative behaviour which may arise within realistic ranges of these parameters.

To this end, we have chosen 'typical' values for the dimensions of the mould (on the basis of thin geometry) and certain properties of the plastic melt, namely, thermal diffusivity, $\kappa$, and shear viscosity, $\mu$.

For fixed aspect ratio $\alpha$ and entry temperature parameter $\beta$, the development of the solid sidewall skin was calculated for variations in the parameters involving the plastic coefficients $\mu$ and $\kappa$, respectively. The results are shown in Figures 2 and 3. For the figures in this section, actual values for $\mu$ and $\kappa$ are chosen, based on the properties of industrial plastics, specifically low-density polyethylene [11], [15].

The situation is completely determined by assigning values to $Q, \alpha, \beta$ and

$$
k_{1}=\frac{k}{\rho c}, \quad k_{2}=\frac{\mu_{0}}{k_{s} H_{0}^{2}\left(T_{s}-T_{w}\right)}, \quad k_{3}=\frac{\mu}{H_{0}^{3}}
$$

since

$$
\eta=k_{1} / Q, \quad \gamma=k_{2} Q^{2}, \quad P_{0}=k_{3} Q_{0}
$$

Figure 5 displays the thickness of the channel for various flux values, and is consistent with the behaviour predicted by Figures 2 and 3. Larger values of flux correspond to higher velocities, which means less heat is actually lost in travelling a certain distance along the mould, resulting in a thinner plastic layer. 


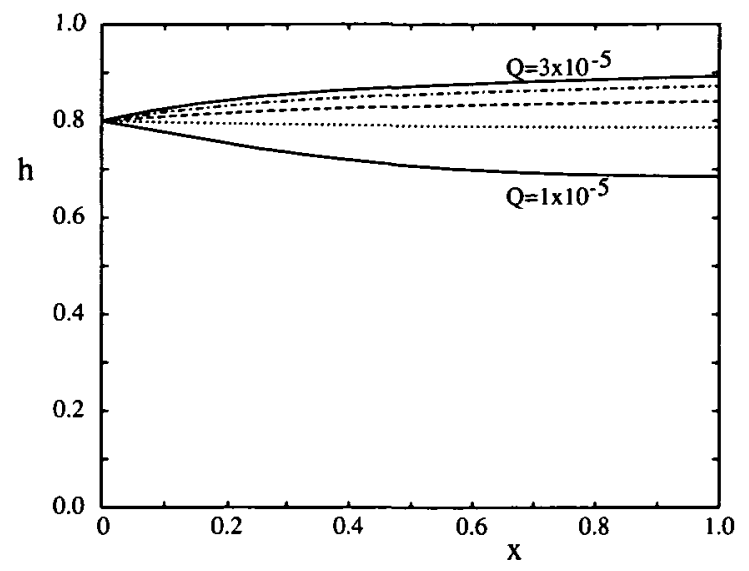

FIGURE 5. Development of the solid plastic skin thickness for variations in the flux $Q$ for a particular 'experiment'. Here, $k_{1}=10^{-7}, k_{2}=10^{10}, \alpha=200$, and $\beta=0.25$. $Q$ values increase uniformly from $1 \times 10^{-5}$ to $3 \times 10^{-5}$.

We also notice that if the filling rate is sufficiently high the channel actually opens. It starts at some finite thickness and the fluid is being forced through a narrow gap. In circumstances where there are high filling rates, so much heat is generated by viscous effects that the fluid actually heats up and the channel widens. A quick calculation reveals that the channel will initially widen if

$$
\gamma>h_{0}^{3} /\left(1-h_{0}\right)
$$

Figure 6 shows the influence of solidification on the pressure versus flow rate curves for various entry temperatures $T_{0}$. The isothermal curve depicts the flux-pressure relationship in a mould where the plastic melt remains at the same temperature for the duration of the filling time and consequently no solidification takes place. From Figure 6 we see that the isothermal pressure falls steadily with decreasing flow rate. Moving up through the curves is equivalent to a decrease in the injection temperature.

When the injection temperature is high, the curves approximate the isothermal case quite closely. However as the injection temperature is reduced, the plastic is more prone to solidification within the length of the mould, and the curve deviates from the isothermal case. A greater pressure gradient is then required along the length of the mould to push the melt through the restricted space.

For each curve there is a minimum pressure drop, $P_{0 \text { min }}$, beneath which flow will not be maintained. Note that for each value of the pressure drop, there are two possible values of the flux. It is likely that one of these is stable, and the other unstable. Suppose we are at the minimum pressure drop, $P_{0 \text { min }}$, for a certain situation, and we increase 


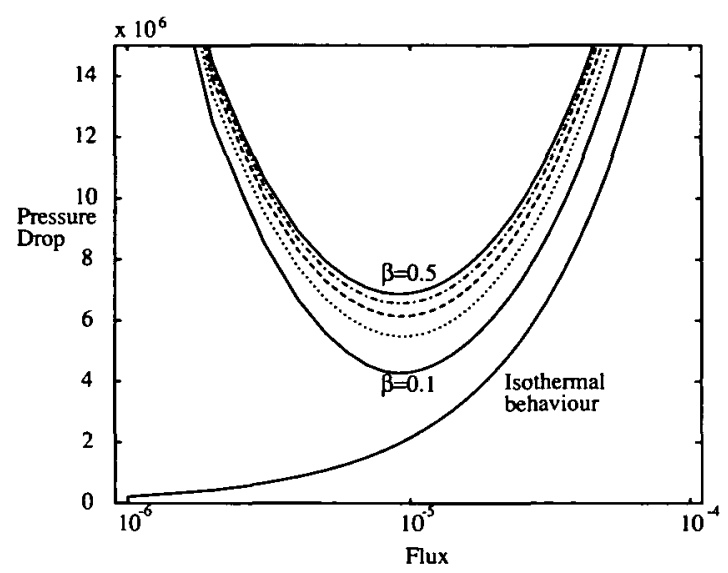

FIGURE 6. Plot of flux against pressure drop for different entry temperatures for the parameters used in the flows depicted in Figure 5. The isothermal case corresponds to the case in which no skin develops. Here, $k_{1}=10^{-7}, k_{2}=10^{10}, k_{3}=2.16 \times 10^{11}, \alpha=200$, and $\beta$ ranges from $\beta=0.1$ to $\beta=0.5$ in steps of size 0.1 .

the applied pressure difference by a small amount. One would expect that this would lead to an increase in the flux, and consequently the state of the system would move to the right along the curve. This suggests that perhaps the left branch of the curve, with the lower flux, is an unstable branch. In turn, this means that the actual minimum flux is that which occurs at the minimum pressure drop. Several numerical experiments were performed using the fixed pressure drop approach, that is, simultaneously solving (4.5) and (4.9) subject to (3.4). In every case the flux obtained corresponded to the right hand branch of Figure 6 , supporting the hypothesis that this is the stable branch.

\section{Conclusion}

As a preliminary investigation of the injection moulding process, we have developed a steady state model that describes the behaviour of a Newtonian fluid in a mould of simple geometry during the mould-filling stage. Using the assumptions of lubrication theory, and the assumption that the velocity and temperature profiles retain their shape as they move through the mould, leads to a pair of coupled equations. If the flux through the mould is fixed, the equations decouple into a single first order nonlinear equation for the thickness of the skin of solid plastic which forms along the walls of the mould.

Numerical solutions of this equation give two branches of solution for a fixed pressure drop in the mould, and indications are that only one of these branches is 
stable. This means that there is a minimum pressure drop along the mould for a given situation, beneath which no flow will be generated and the mould will close off. This corresponds to a minimum in the flux through the mould. The behaviour of the plastic melt was covered in a range of situations, including different lengths of mould, plastic properties and injection temperatures and this same behaviour was evident. The flux-pressure relationship in the mould was compared to that which would occur if no solidification took place, with consistent results.

Clearly there are inadequacies associated with this model. It cannot be used when we expect the profile shape to change. For instance, in the 'thermal runaway' situation [8], or in the case of 'hot core flow' where a thin jet of hot fluid penetrates a long way down the centre of the channel [9].

However, it has been the aim throughout to develop a qualitative understanding of the behaviour of the melt in the injection moulding process with a minimum of mathematical complexity. It has shown some very interesting behaviour which is worthy of further investigation.

As a next step, the model could be used to examine the temperature-dependent viscosity case with only slight extensions. Polymer melts are essentially non-Newtonian visco-elastic fluids, and it is to be expected that inclusion of this dependence would produce some interesting variations on the results obtained in this paper.

\section{References}

[1] C. Austin and A. Miller, "Problem 6. Two phase flow through a plastic mould", in Proc. of the 1986 Math. in Industry Study Group (ed. F. de Hoog), CSIRO Division of Mathematics and Statistics, (1987).

[2] B. S. Chen and W. H. Liu, "Numerical simulation and experimental investigation of injection mold filling with melt solidification", Polymer Eng. and Sci. 29 (1989) 1039-1050.

[3] A. Couniot and M. J. Crochet, "Finite elements for the numerical simulation of injection molding", Proc. 2nd Int. Conf. on Numer. Methods in Industrial Forming Processes (A. A. Balkema, 1986) 165-170.

[4] C. M. Elliot and J. R. Ockendon, Weak and variational methods for moving boundary problems (Pitman, 1982).

[5] A. A. Flaman, R. de Groot and B. Veltman, "Injection moulding experiments ; a challenge to numerical simulation programmes", Plastics and rubber processing and appl. 10 (1988) 155-163.

[6] Y. Kuo and M. Kamal, "Flow of thermoplastics in the filling and packing stages of injection moulding", in Science and Technology of polymer processing : Proc. Int. Conf. on Polymer Processing held at MIT August 1977 (eds N. Suh and N. Sung), (MIT Press, 1979).

[7] L. Laskowski and N. Stokes, "Problem 7. Warping of moulded plastics", in Proc. 1986 Mathematics in Industry Study Group (ed. F. de Hoog), CSIRO Division of Mathematics and Statistics, (1987).

[8] H. Ockendon, "Channel flow with temperature dependent viscosity and intemal viscous dissipation", J. Fluid. Mech. 93 (1979) 737-746.

[9] J. Pearson, "Variable viscosity flows in a channel with high heat generation", J. Fluid. Mech. 83 (1977) 191-206. 
[10] L. Rosenhead, Laminar boundary layers (Clarendon Press, 1963).

[11] I. I. Rubin, Handbook of plastic materials and technology (Wiley, 1990).

[12] A. Tayler, Mathematical models in applied mechanics (Clarendon Press, 1986).

[13] K. Wang, et al., "Cornell injection moulding project", in Science and Technology of polymer processing : Proc. Int. Conf. on Polymer Processing held at MIT August 1977 (eds N. Suh and N. Sung), (MIT Press, 1979).

[14] K. K. Wang, "Computer-aided engineering for injection moulding of plastics", Amer. Inst. of Chem. Eng. Workshop. 84 (AIChE, New York, 1988) 37-51.

[15] T. M. Yarwood and T. G. Francis, Engineering and physical tables (Macmillan, 1963). 